cefixime, ceftriaxone, azithromycin, ciprofloxacin and penicillin were defined according to EUCAST 4.0 standards.

Results Of 2115 isolates, $91.6 \%$ of isolates were from men. The most frequently tested materials among men were urethral (92.4\%) and rectal swabs (3.8\%), and among women mainly endocervical swabs (80.9\%). Resistance to ceftriaxone (MIC $\geq 0.125 \mathrm{mg} / \mathrm{L}$ ) occurred only sporadically $(0-0.3 \%)$ during the entire observation period (2015 and 2018), while 1.0$2.1 \%$ of isolates were resistant to cefixime (MIC $\geq 0.125 \mathrm{mg} /$ L). Proportion of isolates resistant to azithromycin (MIC $\geq$ $0.5 \mathrm{mg} / \mathrm{L})$ was $11.4 \%$ (2014), $11.3 \%$ (2015), 4.3\% (2016), $3.7 \%$ (2017), 9.4\% (2018). $53.4-71.7 \%$ were resistant to ciprofloxacin, and 14.2-24.3.1\% were resistant to penicillin.

Conclusion Resistance to ceftriaxone and to cefixime was low, whereas azithromycin resistance showed a discontinuous presentation with partly high levels during the observation period. Rates of ciprofloxacin and penicillin resistance were very high. According to the current national guidelines, ceftriaxone 1-2g IV and azithromycin 1,5g orally are usually used in dual therapy. Shortly revised national guidelines will state that use of azithromycin should be avoided if possible if a test of cure can be guaranteed and a susceptibility test is available. Continued surveillance of NG AMR remains relevant to ensure efficient disease management.

Disclosure No significant relationships.

\section{P672 GENETIC PATHWAY TO HIGH LEVEL AZITHROMYCIN RESISTANCE IN NEISSERIA GONORRHOEAE}

${ }^{1}$ Els Verhoeven, ${ }^{1}$ Saïd Abdellati*, ${ }^{1}$ Irith De Baetselier, ${ }^{2}$ Tania Crucitti, ${ }^{3}$ Chris Kenyon. ${ }^{1}$ Institute of Tropical Medicine, Clinical Sciences, Antwerp, Belgium; ${ }^{2}$ Centre Pasteur $d u$ Cameroun, Yaoundé, Cameroon; ${ }^{3}$ Institute of Tropical Medicine, HIVISTI Unit, Antwerp, Belgium

\subsection{6/sextrans-2019-sti.739}

Background The in-vitro genetic pathways to high-level azithromycin (AZM) resistance have hitherto not been established in Neisseria gonorrhoeae (Ng).

Methods A Ng morbidostat that dynamically increases AZM concentrations in response to $\mathrm{Ng}$ growth was built according to the protocol of Toprak et al. The reference strains $\mathrm{Ng}$ WHO-F and WHO-X were grown in $12 \mathrm{~mL}$ GC broth supplemented with IsoVitaleX ${ }^{\mathrm{TM}}(1 \%)$ and vancomycin, colistin, nystatin, trimethoprim selective supplement for 30 days in a $6 \% \mathrm{CO}_{2}$ environment at $36^{\circ} \mathrm{C}$. Depending on the turbidity and growth of the culture, $1 \mathrm{~mL}$ of fresh medium or AZM was added to the culture vials after each cycle of 21 minutes. The experiment started with a concentration of $20 \mathrm{x}$ minimal inhibitory concentration (MIC) of AZM in the drug reservoir which was increased up to $320 \mathrm{x}$ MIC for both strains by the end of the experiment. Samples of the cultures were taken 23 times a week and MICs of AZM were determined using Etests. Whole genome sequencing will be performed using Illumina MiSeq. All experiments were run in triplicate.

Results The initial MICs of WHO-F and WHO-X were 0,125 $\mu \mathrm{g} / \mathrm{mL}$ and $0,25 \mu \mathrm{g} / \mathrm{mL}$ respectively. In the first week, the MICs of WHO-F and WHO-X increased approximately 24fold for WHO-F and 48-fold for WHO-X. After 30 days, WHO-F and WHO-X had attained MICs of $96 \mu \mathrm{g} / \mathrm{mL}$ and $\geq 296 \mu \mathrm{g} / \mathrm{mL}$, respectively. The genetic pathways to resistance will be analysed and presented.
Conclusion We were able to induce high level AZM resistance in $\mathrm{Ng}$ within 30 days of AZM exposure using our $\mathrm{Ng}$ morbidostat.

Disclosure No significant relationships.

\section{P673 IN-VITRO ACTIVITY OF SMT-571 AND COMPARATORS AGAINST CLINICAL ISOLATES AND REFERENCE STRAINS OF NEISSERIA GONORRHOEAE}

${ }^{1}$ Paul Meo*, ${ }^{1}$ Clive Mason, ${ }^{1}$ Nawaz Khan, ${ }^{2}$ Magnus Unemo, ${ }^{2}$ Susanne Jacobsson. ${ }^{1}$ Summit Therapeutics, Cambridge, UK; ${ }^{2}$ Örebro University Hospital, Örebro, Sweden

\subsection{6/sextrans-2019-sti.740}

Background The emergence and spread of multidrug resistance to antibiotics used to treat gonorrhoea has resulted in a dramatic loss of effective regimens for the condition. Currently, the extended spectrum cephalosporin, ceftriaxone, is the only viable monotherapy option available, however, resistance to this last line treatment is now emerging globally. Herein, we assessed the in vitro activity of a novel small molecule antimicrobial with a new mechanism of action, SMT-571, against a large collection of $N$. gonorrhoeae clinical isolates and reference strains including numerous MDR and XDR gonococcal isolates.

Methods MICs (mg/L) of SMT-571 were determined by agar dilution according to current CLSI guidelines. The MICs of ceftriaxone, cefixime, azithromycin, ciprofloxacin, spectinomycin, tetracycline, and ampicillin were determined using the Etest method (AB bioMérieux, Marcy l'Etoile, France).

Results SMT-571 showed potent in vitro activity against all the tested $N$. gonorrhoeae isolates $(n=262)$ with MICs ranging from 0.064 to $0.125 \mathrm{mg} / \mathrm{L}$, and the MIC50, MIC90 and modal MIC were all $0.125 \mathrm{mg} / \mathrm{L}$. The compound was not influenced by pre-existing resistance mechanisms with no cross-resistance or correlation between the MICs of SMT-571 and comparator agents being observed.

Conclusion This study is the first broad evaluation of the in vitro activities of a new mechanism, novel small molecule antimicrobial for the treatment of gonorrhoea. SMT-571 demonstrated high in vitro activity against a large geographically, temporally and genetically diverse collection of clinical $N$. gonorrhoeae isolates and international reference strains, including various types of high-level resistant, MDR and XDR gonococcal isolates.

Disclosure No significant relationships.

\section{P675 TWO RECENT CASES OF EXTENSIVELY DRUG-RESISTANT (XDR) GONORRHOEA IN THE UNITED KINGDOM LINKED TO A EUROPEAN PARTY DESTINATION}

${ }^{1}$ Paddy Horner*, ${ }^{1}$ Helen Fifer, ${ }^{1}$ Bavithra Nathan, ${ }^{2}$ David Eyre, ${ }^{1}$ Katy Town, ${ }^{1}$ Hamish Mohammed, ${ }^{1}$ Michelle Cole, ${ }^{1}$ Rachel Pitt, ${ }^{1}$ Maya Gobin, ${ }^{1}$ Charles Irish, ${ }^{1}$ Daniel Gardiner, ${ }^{1}$ James Sedgwick, ${ }^{1}$ Charles Beck, ${ }^{1}$ John Saunders, ${ }^{1}$ Deborah Turbitt, ${ }^{1}$ Clare Cook, ${ }^{2}$ Teresa Street, ${ }^{2}$ Leanne Baker, ${ }^{2}$ Nicholas Sanderson, ${ }^{1}$ Nick Phin. ${ }^{1}$ PHE and BASHH, Incident Management Team, London, UK; '2University of Oxford, Nuffield Department of Medicine, Oxford, UK

\subsection{6/sextrans-2019-sti.741}

Background The development of antimicrobial resistance (AMR) to macrolides and extended-spectrum cephalosporins (ESC) in Neisseria gonorrhoeae (NG), is a major public health 
concern. While macrolide AMR is increasing globally, ESC AMR is low and extensive drug-resistance (XDR) rare.

Methods We report the clinical management and microbiology of two recent cases of XDR NG in the UK.

Results Case A (female) presented to a sexual health clinic (SHC) in October 2018 with urinary symptoms. She cleared the infection following treatment with ceftriaxone $500 \mathrm{mg}$ plus azithromycin $1 \mathrm{~g}$. She had recently had vaginal intercourse with $\geq 1$ male partner in Ibiza, Spain. Case B (female) presented to a SHC elsewhere in England in November 2018 with anal and genital symptoms. She had recently had unprotected vaginal, oral and anal sex with an asymptomatic man who had been in Ibiza and had links with the same sexual network as case A. He tested NG NAAT-negative in December without treatment. Case B initially responded clinically to treatment with ceftriaxone $1 \mathrm{~g}$, but symptoms relapsed and she remained culture-positive 2 weeks later. She failed subsequent treatment with gentamicin $240 \mathrm{mg}$ plus azithromycin $2 \mathrm{~g}$ but cleared the infection with 3 days of IV ertapenem. Both isolates were resistant to ceftriaxone (MIC $1.0 \mathrm{mg} / \mathrm{L}$ ), cefixime, penicillin, ciprofloxacin and tetracycline, had intermediate susceptibility to azithromycin (MIC $0.5 \mathrm{mg} / \mathrm{L}$ ), and were susceptible to spectinomycin. Whole genome sequencing indicated that both isolates were from the FC428 clone, which has been reported sporadically globally, usually with epidemiological links to the Asia-Pacific region.

Conclusion These FC428 clone isolates are able to cause both asymptomatic and symptomatic infection with a variable response to ceftriaxone and azithromycin treatment. This clone is likely to increase over time in Europe and threatens the effectiveness of gonorrhoea treatment. Surveillance of AMR, test-of-cure, extra-genital sampling and partner notification are vital to maintain effective treatment and prevent spread.

Disclosure No significant relationships.

\section{P676 AZITHROMYCIN RESISTANCE AMONG NEISSERIA GONORRHOEAE CASES IN KING COUNTY, WASHINGTON, USA, 2017-2018}

${ }^{1}$ Christina Thibault*, ${ }^{2}$ Olusegun Soge, ${ }^{3}$ Lindley Barbee, ${ }^{1}$ Roxanne Kerani, ${ }^{1}$ Dawn Spellman, ${ }^{1}$ Sarah Stewart, ${ }^{1}$ Anna Harrington, ${ }^{2}$ Rushlenne Pascual, ${ }^{3}$ Matthew Golden. ${ }^{1}$ Public Health Seattle and King County, HIVISTD Program, Seattle, USA; ${ }^{2}$ University of Washington, Global Health, Seattle, USA; ${ }^{3}$ University of Washington, Medicine, Seattle, USA

\subsection{6/sextrans-2019-sti.742}

Background Public Health - Seattle \& King County (PHSKC) participates in Strengthening the U.S. Response to Resistant Gonorrhea (SURRG), funded by the Centers for Disease Control and Prevention, to enhance surveillance of antimicrobial resistance in Neisseria gonorrhoeae (GC). We aimed to identify predictors of azithromycin-resistant GC.

Methods GC culture specimens were collected from patients with GC infection who attended the PHSKC STD Clinic or one of five King County, Washington, medical clinics during 2017-2018. Positive culture isolates underwent azithromycin susceptibility testing with Etest; azithromycin resistance (AZI$\mathrm{R})$ was defined as minimum inhibitory concentration $\geq 2.0 \mu \mathrm{g} /$ $\mathrm{mL}$. Clinical and sociobehavioral data was obtained from medical records and partner services interviews; we compared characteristics of cases with and without AZI-R and evaluated statistical significance with chi-square and Fisher's exact tests. Results During 2017-2018, 1,164 GC isolates from 1,048 unique cases underwent azithromycin Etest susceptibility testing. Overall, $6.5 \%$ of isolates and $6.8 \%$ of cases had AZI-R. Prevalence was higher in rectal isolates $(8.3 \%$ of 373$)$ than urethral $(5.0 \%$ of $496, \mathrm{p}=0.05)$ and similar to pharyngeal (7.4\% of $270, p=0.68)$. AZI-R was more common in men who have sex with men (MSM) than heterosexuals ( $8 \%$ vs $3 \%, \mathrm{p}=0.04)$, and in Hispanics vs. non-Hispanics $(12 \%$ vs $6 \%, \mathrm{p}<0.01)$. Age, GC history, number of sex partners, HIVstatus, PrEP-status, and diagnosing facility type were not associated with AZI-R. Most (95\%) AZI-R cases were treated with an azithromycin-containing regimen (dual therapy with ceftriaxone (92\%) or gentamicin (4\%)). Nearly all (96\%) had a documented negative test of cure (culture and/or NAAT); no treatment failures were observed.

Conclusion AZI-R was identified in $6.8 \%$ of GC cases in King County, Washington. While more common in MSM and Hispanic persons, AZI-R was not strongly associated with other characteristics we evaluated. Health care providers should be aware of the potential for AZI-R in patients with GC.

Disclosure No significant relationships.

\section{P677 PRESCRIBED TREATMENTS FOR NEISSERIA GONORRHOEAE INFECTIONS AND TREATMENT FAILURES IN THE QUEBEC SENTINEL NETWORK, 2015- 2017}

${ }^{1}$ Fannie Defay*, ${ }^{2}$ Karine Blouin, ${ }^{3}$ Sylvie Venne, ${ }^{4}$ Brigitte Lefebvre, ${ }^{5}$ Annick Trudelle, ${ }^{6}$ AnnieClaude Labbe. ${ }^{1}$ Institut National de Santé Publique, Infections Transmissibles Sexuellement et par le Sang, Montréal, Canada; ${ }^{2}$ Institut National de Santé Publique du Québec, Infections Transmissibles Sexuellement et par le Sang, Québec, Canada; ${ }^{3}$ Quebec Health Ministry, Public Health Direction, STD Prevention Direction, Montréal, Canada; ${ }^{4}$ Laboratoire Santé Publique du Québec, Montréal, Canada; ${ }^{5}$ nstitut National de Santé Publique, Montréal, Canada; ${ }^{6}$ Montreal University, Departement de Microbiologie Infectiologie, Montreal, Canada

\subsection{6/sextrans-2019-sti.743}

Background Neisseria gonorrhoeae is becoming increasingly resistant to the antibiotics used and many countries reported therapeutic failures. The WHO published a global action plan on antimicrobial resistance. Several measures were undertaken in Québec including the use of a dual therapy. Since 2015, the provincial sentinel network aims to 1) maintain sufficient cultures for the surveillance of antimicrobial resistance 2) complement the reference laboratory antimicrobial surveillance by providing epidemiological and clinical information and 3) complement the surveillance of treatment failures.

Methods Two clinics specialized in STBBIs and several general practitioners, located in three regions (Montreal, Montérégie, Nunavik) are enrolled. Epidemiological and clinical data (including reasons for visits, laboratory samples and treatments prescribed) of gonococcal infections are collected on a centralized secured web application. Cases are classified as retained treatment failures (presence of all predefined criteria) or suspected treatment failures.

Results From September 2015 to December 2017, 1240 episodes in 1115 individuals were recorded (111 women, 1000 men, 3 transgender, 1 unknown sex). At least $83 \%$ of the 